\title{
On Hecke $L$-functions associated with cusp forms
}

\author{
by
}

\section{A. Sankaranarayanan (Mumbai)}

1. Introduction. Let $f(z)=\sum_{n=1}^{\infty} a_{n} e^{2 \pi i n z}$ be a holomorphic cusp form of even integral weight $k>0$ with respect to the modular group $\Gamma=$ $\mathrm{SL}(2, \mathbb{Z})$ and define the associated Hecke $L$-function by

$$
L_{f}(s)=\sum_{n=1}^{\infty} a_{n} n^{-s}
$$

for $\Re s>(k+1) / 2$. Throughout this paper we assume that $f(z)$ is a Hecke eigenform with $a_{1}=1$. It is known (see [7]) that $L_{f}(s)$ admits analytic continuation to $\mathbb{C}$ as an entire function and it satisfies the functional equation

$$
(2 \pi)^{-s} \Gamma(s) L_{f}(s)=(-1)^{k / 2}(2 \pi)^{-(k-s)} \Gamma(k-s) L_{f}(k-s) .
$$

$L_{f}(s)$ has an Euler product representation (for $\Re s>(k+1) / 2$ )

$$
L_{f}(s)=\prod_{p}\left(1-a_{p} p^{-s}+p^{k-1} p^{-2 s}\right)^{-1} .
$$

The non-trivial zeros of $L_{f}(s)$ lie within the critical strip $(k-1) / 2<\Re s<$ $(k+1) / 2$, symmetrically to the real axis and also to the line $\Re s=k / 2$. The Riemann hypothesis in this situation asserts that all non-trivial zeros are on the critical line $\Re s=k / 2$. From Deligne's proof of Ramanujan-Peterson's conjecture (see [2] and [3]), we have the bound for the coefficients

$$
\left|a_{n}\right| \leq d(n) n^{(k-1) / 2} .
$$

We denote by $N_{f}(T)$ the number of zeros $\beta+i \gamma$ of $L_{f}(s)$ for which $0<\gamma<T$, for $T$ not equal to any $\gamma$; otherwise we put

$$
N_{f}(T)=\lim _{\varepsilon \rightarrow 0} \frac{1}{2}\left\{N_{f}(T+\varepsilon)+N_{f}(T-\varepsilon)\right\} .
$$

2000 Mathematics Subject Classification: Primary 11F66; Secondary 11F11, 11M41.

Key words and phrases: holomorphic cuspform, Hecke $L$-functions, density estimates in the neighbourhood of the critical line. 
Then one can show that (following Theorem 9.3 of [14])

$$
N_{f}(T)=\frac{T}{\pi} \log \frac{T}{\pi}-\frac{T}{\pi}+1+S_{f}(T)+O(1 / T)
$$

where

$$
S_{f}(t)=\frac{1}{\pi} \arg L_{f}(k / 2+i t) .
$$

The amplitude is obtained by a continuous variation along the straight lines joining the points $k / 2+1, k / 2+1+i T$ and $k / 2+i T$, starting with the value zero. Hence the variation of $S_{f}(t)$ is closely connected with the distribution of the imaginary parts of the zeros of $L_{f}(s)$.

We now define, for $\sigma \geq k / 2, T \geq 1$ and $H \leq T$,

$$
N_{f}(\sigma, T, T+H)=\left|\left\{\beta+i \gamma: L_{f}(\beta+i \gamma)=0, \beta \geq \sigma, T \leq \gamma \leq T+H\right\}\right| .
$$

\section{Notation and preliminaries}

- $A_{1}, A_{2}, \ldots$ denote effective absolute constants, sometimes positive.

- $f(x) \ll g(x)$ and $f(x)=O(g(x))$ will mean that there exists a constant $C>0$ such that $|f(x)| \leq C g(x)$.

- $\varepsilon$ denotes any small positive constant.

- As usual, $s=\sigma+i t, w=u+i v$.

When $k$ is even, it is known that $a_{n}$ 's are real and in fact they are totally real algebraic numbers. Hence $a_{p}$ is real from (1.1) and (1.3). By Deligne's estimate, we also have $\left|a_{p}\right| \leq 2 p^{(k-1) / 2}$. We define a real number $A_{p}^{\prime}$ such that $a_{p}=2 A_{p}^{\prime} p^{(k-1) / 2}$ and clearly $\left|A_{p}^{\prime}\right| \leq 1$. Let $\alpha_{p}^{\prime}$ and $\bar{\alpha}_{p}^{\prime}$ be the roots of the equation $x^{2}-2 A_{p}^{\prime} x+1=0$; note that $\left|\alpha_{p}^{\prime}\right|=1$. Therefore, from the Euler product of $L_{f}(s)$, we can write

$$
L_{f}(s)=\prod_{p}\left(1-\alpha_{p} p^{-s}\right)^{-1}\left(1-\bar{\alpha}_{p} p^{-s}\right)^{-1}
$$

with $\left|\alpha_{p}\right|=p^{(k-1) / 2}$ and $a_{p}=\alpha_{p}+\bar{\alpha}_{p}$. Taking logarithms and differentiating both sides with respect to $s$ we find that

$$
-\frac{L_{f}^{\prime}}{L_{f}}(s)=\sum_{m \geq 1, p}\left(\alpha_{p}^{m}+\bar{\alpha}_{p}^{m}\right) p^{-m s}(\log p) .
$$

Now we define

$$
\Lambda_{f}(n)= \begin{cases}\left(\alpha_{p}^{m}+\bar{\alpha}_{p}^{m}\right)(\log p) & \text { if } n=p^{m} \\ 0 & \text { otherwise. }\end{cases}
$$

Hence we obtain

$$
-\frac{L_{f}^{\prime}}{L_{f}}(s)=\sum_{n=2}^{\infty} \Lambda_{f}(n) n^{-s} \quad(\text { in } \Re s>(k+1) / 2) .
$$


Note that

$$
\Lambda_{f}(n) \leq 2(\log n) n^{(k-1) / 2}
$$

Let $x>1$ and write

(2.6) $\Lambda_{x, f}(n)$

$$
= \begin{cases}\Lambda_{f}(n) & \text { if } 1 \leq n \leq x, \\ \Lambda_{f}(n) \frac{\left\{\left(\log \left(x^{3} / n\right)\right)^{2}-2\left(\log \left(x^{2} / n\right)\right)^{2}\right\}}{2(\log x)^{2}} & \text { if } x \leq n \leq x^{2}, \\ \Lambda_{f}(n) \frac{\left(\log \left(x^{3} / n\right)\right)^{2}}{2(\log x)^{2}} & \text { if } x^{2} \leq n \leq x^{3} .\end{cases}
$$

We define a non-negative smooth $C^{\infty}$ function $\Psi_{U}(t)$ as follows. For $H \leq T$,

$$
\Psi_{U}(t)= \begin{cases}0 & \text { if } t<1+1 / U \text { or } t>1+H / T-1 / U \\ 1 & \text { if } 1+1 / U \leq t \leq 1+H / T-1 / U\end{cases}
$$

Also assume that $\Psi_{U}$ is chosen in such a way that

$$
\Psi_{U}^{(p)}(t) \ll U^{p}
$$

where $U$ is a positive parameter to be fixed later. Let $\phi(\xi), \phi^{*}(\xi)$ be suitable smooth $\left(\mathcal{C}^{\infty}\right)$ functions satisfying $\phi^{*}(\xi)=1-\phi(1 / \xi)$ and

$$
\phi(\xi)= \begin{cases}1 & \text { if }|\xi| \leq 2 / 3 \\ 0 & \text { if }|\xi| \geq 3 / 2\end{cases}
$$

Define

$$
L_{f}^{-1}(s)=\sum_{n=1}^{\infty} \mu_{f}(n) n^{-s} \quad \text { in } \Re s>(k+1) / 2,
$$

so that from the Euler product for $L_{f}(s)$, we have

Now, we define

$$
\mu_{f}\left(p^{r}\right)= \begin{cases}1 & \text { if } r=0 \\ -a_{p} & \text { if } r=1 \\ p^{k-1} & \text { if } r=2 \\ 0 & \text { if } r \geq 3\end{cases}
$$

$$
g_{\xi}(n)= \begin{cases}1 & \text { if } 1 \leq n \leq \xi \\ \frac{\log \left(\xi^{2} / n\right)}{\log \xi} & \text { if } \xi \leq n \leq \xi^{2} \\ 0 & \text { if } n \geq \xi^{2}\end{cases}
$$

and define $\lambda_{n}=\mu_{f}(n) g_{\xi}(n)$. Here $\xi=T^{\theta}$ with $0<\theta<1 / 4$ to be chosen appropriately later. We introduce a Dirichlet polynomial as in [9],

$$
M_{\xi^{2}}(s)=\sum_{v=1}^{\infty} \lambda_{v} v^{-s}
$$


In this paper we prove the following two theorems.

TheOREm 1. For $t \geq 2,2 \leq x \leq t^{2}$, we have

$$
\begin{aligned}
S_{f}(t)= & -\frac{1}{\pi} \sum_{n<x^{3}} \frac{\Lambda_{x, f}(n) \sin (t \log n)}{n^{\sigma_{x, t}} \log n}+O\left(\left(\sigma_{x, t}-k / 2\right)\left|\sum_{n<x^{3}} \frac{\Lambda_{x, f}(n)}{n^{\sigma_{x, t}+i t}}\right|\right) \\
& +O\left(\left(\sigma_{x, t}-k / 2\right) \log t\right),
\end{aligned}
$$

where

$$
\sigma_{x, t}=k / 2+2 \max (\beta-k / 2,2 / \log x)
$$

with $\varrho=\beta+i \gamma$ running over those zeros for which

$$
|t-\gamma| \leq x^{3|\beta-k / 2|}(\log x)^{-1}
$$

and $\Lambda_{x, f}(n)$ is as in (2.6).

As corollaries, by choosing $x=\sqrt{\log t}$ we obtain

$$
S_{f}(t)=O(\log t)
$$

unconditionally, and assuming Riemann hypothesis, we get

$$
S_{f}(t)=O\left(\frac{\log t}{\log \log t}\right) .
$$

TheOREM 2. Let B be any fixed small positive constant. Let

$$
B^{\prime}=\frac{19}{20}+\frac{13.505}{5} B \quad \text { and } \quad B^{\prime}<\alpha \leq 1 .
$$

Then for $T^{\alpha} \leq H \leq T$, we have

$$
N_{f}(\sigma, T, T+H) \ll H\left(\frac{H}{T^{B^{\prime}}}\right)^{-\frac{B}{1-B^{\prime}}(\sigma-k / 2)} \log T
$$

uniformly for $k / 2 \leq \sigma \leq(k+1) / 2$.

REMARK 1. Theorems 1 and 2 (with $T^{1 / 2+\varepsilon} \leq H \leq T$ and $B^{\prime}=1 / 2$ ) in the case of the Riemann zeta-function $\zeta(s)$ are due to Selberg [13]. The importance of Theorem 2 is in the exponent of the $\log$ factor when $|\sigma-k / 2|$ $\ll(\log T)^{-1}$. In fact later developments in the theory allow us to take even a much shorter interval in the case of $\zeta(s)$ in Theorem 2. Theorem 2 (with $H=T)$ in the case of $L_{f}(s)$ is due to Luo [9]. Here we prove an analogue of a result of Selberg for $L_{f}(s)$ (Theorem 1) and the density estimate for $L_{f}(s)$ over shorter intervals (Theorem 2 ). We follow closely the papers [13] and [9].

REMARK 2. It should be pointed out here that some more important results have recently been proved in [1] assuming certain hypotheses (which are true in this situation) for a class of Dirichlet series which are linear combinations of Euler products. We also suggest some basic references related to our paper: [6], [8], [11], [12]. 
Acknowledgements. The author is highly indebted to the referee for making some useful comments.

\section{Some lemmas}

LEMma 3.1. We have $\xi(s)=(2 \pi)^{-s} \Gamma(s) L_{f}(s)$ is an integral function of order 1 .

Proof. It is standard.

Lemma 3.2. If $s \neq \varrho, t \geq 2$, then

$$
\frac{L_{f}^{\prime}}{L_{f}}(s)=\sum_{\varrho}\left((s-\varrho)^{-1}+\varrho^{-1}\right)+O(\log t)
$$

uniformly for $k / 2 \leq \sigma \leq k / 2+10$.

Proof. Since $\xi(s)$ is an integral function of order 1 it has the Weierstrass product representation

$$
\xi(s)=e^{b_{0}+b_{1} s} \prod_{\varrho}\left\{\left(1-\frac{s}{\varrho}\right) e^{s / \varrho}\right\}
$$

where $b_{0}, b_{1}$ are certain constants. Also we have

$$
\xi(s)=(2 \pi)^{-s} \Gamma(s) L_{f}(s) .
$$

Taking logarithms and differentiating (3.2.1) and (3.2.2) with respect to $s$, and using (for $a \leq \Re z \leq b$ )

$$
\frac{\Gamma^{\prime}}{\Gamma}(z)=\log z-\frac{1}{2 z}+O\left(|z|^{-2}\right),
$$

we obtain the lemma.

Lemma 3.3. In the region defined by $\sigma \leq 1 / 4,|s-n| \geq 1 / 2(n=$ $0,-1,-2, \ldots)$, we have

$$
\left|\frac{L_{f}^{\prime}}{L_{f}}(s)\right|<A_{1}(\log |s|+1) .
$$

Proof. From the functional equation $\xi(s)=e^{i \pi k / 2} \xi(k-s)$, we have

$$
\frac{L_{f}^{\prime}}{L_{f}}(s)=2 \log (2 \pi)-\frac{\Gamma^{\prime}}{\Gamma}(s)-\frac{\Gamma^{\prime}}{\Gamma}(k-s)-\frac{L_{f}^{\prime}}{L_{f}}(k-s) .
$$

Note that, for $\sigma \leq 1 / 4, k-\sigma \geq k-1 / 4$ and hence

$$
\left|\frac{L_{f}^{\prime}}{L_{f}}(k-s)\right| \leq \sum_{n=1}^{\infty} \frac{\Lambda_{f}(n)}{n^{k-\sigma}} \ll 1 .
$$

Now the lemma follows on using (3.2.3). 
LEMmA 3.4. There exists a sequence of numbers $T_{2}, T_{3}, \ldots$ such that $m<T_{m}<m+1(m=2,3, \ldots)$ and

$$
\left|\frac{L_{f}^{\prime}}{L_{f}}(s)\right|<A_{2} \log ^{2} m
$$

for $k / 2+1 \geq \sigma \geq 1 / 4, t= \pm T_{m}$.

Proof. From the Weierstrass product representation of $\xi(s)$, we obtain

$$
\begin{aligned}
\frac{L_{f}^{\prime}}{L_{f}}(s) & =b_{1}+\log (2 \pi)-\frac{\Gamma^{\prime}}{\Gamma}(s)+\sum_{\varrho}\left((s-\varrho)^{-1}+\varrho^{-1}\right) \\
& =g(s)+\Sigma(s) \quad(\text { say })
\end{aligned}
$$

where

$$
g(s)=b_{1}+\log (2 \pi)-\frac{\Gamma^{\prime}}{\Gamma}(s), \quad \Sigma(s)=\sum_{\varrho}\left((s-\varrho)^{-1}+\varrho^{-1}\right) .
$$

Let $s=\sigma+i t, s_{0}=k / 2+2+i t$ where $1 / 4 \leq \sigma \leq k / 2+2, t>2$ and $t$ is not equal to any $\gamma$. Let $\delta_{0}$ be the distance of $t$ from the nearest $\gamma$ and let

$$
\delta=\delta(t)=\min \left(\delta_{0}, 1\right) .
$$

Then for every zero $\varrho=\beta+i \gamma$ with $0 \leq \beta \leq(k+1) / 2$, we have

$$
|s-\varrho|^{2} \geq(t-\gamma)^{2} \geq \delta^{2} / 2+(t-\gamma)^{2} / 2 \geq \frac{\delta^{2}}{2}\left\{1+(t-\gamma)^{2}\right\}
$$

and

$$
\left|s_{0}-\varrho\right|^{2}=(k / 2+2-\beta)^{2}+(t-\gamma)^{2} \geq 1+(t-\gamma)^{2} .
$$

Therefore from (3.4.3) and (3.4.4), we get

$$
\begin{aligned}
\left|\Sigma(s)-\Sigma\left(s_{0}\right)\right| & =\left|\sum_{\varrho} \frac{s_{0}-s}{(s-\varrho)\left(s_{0}-\varrho\right)}\right| \\
& \leq \sum_{\varrho} \frac{k / 2+2-1 / 4}{\left(\delta^{2} / 2\right)^{1 / 2}\left\{1+(t-\gamma)^{2}\right\}} .
\end{aligned}
$$

On the other hand,

$$
\begin{aligned}
\Re \Sigma\left(s_{0}\right) & =\sum_{\varrho}\left(\frac{k / 2+2-\beta}{\left|s_{0}-\varrho\right|^{2}}+\frac{\beta}{|\varrho|^{2}}\right) \\
& \geq \sum_{\varrho}\left\{(k / 2+2-\beta)^{2}+(t-\gamma)^{2}\right\}^{-1} \\
& \geq(k / 2+2)^{-2} \sum_{\varrho}\left\{1+(t-\gamma)^{2}\right\}^{-1} .
\end{aligned}
$$


Hence from (3.4.5) and (3.4.6), we get

$$
\left|\Sigma(s)-\Sigma\left(s_{0}\right)\right|<2 \delta^{-1}(k / 2+2)^{2}(k / 2+2-1 / 4) \Re \Sigma\left(s_{0}\right) .
$$

This implies that

$$
\begin{aligned}
\left|\frac{L_{f}^{\prime}}{L_{f}}(s)-g(s)\right| & =|\Sigma(s)| \leq \delta^{-1}\left\{2(k / 2+2)^{3}+1\right\}\left|\Sigma\left(s_{0}\right)\right| \\
& \leq A_{3}(k) \delta^{-1}\left|\frac{L_{f}^{\prime}}{L_{f}}\left(s_{0}\right)-g\left(s_{0}\right)\right| .
\end{aligned}
$$

Note that $\left|\frac{L_{f}^{\prime}}{L_{f}}\left(s_{0}\right)\right| \ll 1$. Now applying the asymptotic expression (3.2.3) for $\frac{\Gamma^{\prime}}{\Gamma}(s)$ to $g(s)$ and $g\left(s_{0}\right)$, we get

$$
\left|\frac{L_{f}^{\prime}}{L_{f}}(s)\right|<A_{4}(k) \delta^{-1} \log t .
$$

Now let $m$ be any integer greater than 1 , and $\nu_{m}$ the number of $\varrho$ for which $m<\gamma<m+1$ so that $\nu_{m}=N(m+1)-N(m)<A_{5} \log m$. If we divide the interval $(m, m+1)$ into $\nu_{m}+1$ equal parts, then one subinterval at least will contain no $\gamma$ in its interior. We choose $T_{m}$ to be the midpoint of such an interval. If $t=T_{m}$, then $\delta \geq 1 /\left(2\left(\nu_{m}+1\right)\right)$ and hence

$$
\left|\frac{L_{f}^{\prime}}{L_{f}}(s)\right|<A_{6} \log ^{2} m
$$

which proves the lemma.

LEMma 3.5. There exists a sequence of numbers $T_{2}, T_{3}, \ldots$ such that $m<T_{m}<m+1(m=2,3, \ldots)$ and

$$
\left|\frac{L_{f}^{\prime}}{L_{f}}(s)\right|<A_{7} \log ^{2} m
$$

for $\sigma \geq-m-1 / 2, t= \pm T_{m}$ or $\sigma=-m-1 / 2,|t|<T_{m}$.

Proof. This follows from Lemmas 3.3 and 3.4.

Lemma 3.6. For $s \neq \varrho, s \neq-q(q=0,1,2, \ldots)$, we have

$$
\begin{aligned}
\frac{L_{f}^{\prime}}{L_{f}}(s)= & -\sum_{n<x^{3}} \frac{\Lambda_{x, f}(n)}{n^{s}}+(\log x)^{-2} \sum_{q=0}^{\infty} \frac{x^{-q-s}\left(1-x^{-q-s}\right)^{2}}{(q+s)^{3}} \\
& +(\log x)^{-2} \sum_{\varrho} \frac{x^{\varrho-s}\left(1-x^{\varrho-s}\right)^{2}}{(s-\varrho)^{3}} .
\end{aligned}
$$

Proof. First, we notice that for $\alpha_{1}, y>0$,

$$
\frac{1}{2 \pi i} \int_{\alpha_{1}-i \infty}^{\alpha_{1}+i \infty} \frac{y^{w}}{w^{3}} d w= \begin{cases}(\log y)^{2} / 2 & \text { if } y \geq 1 \\ 0 & \text { if } 0<y \leq 1\end{cases}
$$


Fix $\alpha_{1}=\max (k / 2+1,(k+1) / 2+\sigma)$. From (3.6.1), we obtain

$$
\sum_{n<x^{3}} \frac{\Lambda_{x, f}(n)}{n^{s}}=\frac{1}{2 \pi i(\log x)^{2}} \int_{\alpha_{1}-i \infty}^{\alpha_{1}+i \infty} \frac{x^{w}\left(1-x^{w}\right)^{2}}{w^{3}}\left(-\frac{L_{f}^{\prime}}{L_{f}}(s+w)\right) d w .
$$

After taking into account the residues at singularities between the contours, we replace the interval $\left(\alpha_{1}-i T_{m}, \alpha_{1}+i T_{m}\right)$ of the integration line by the straight lines joining the points $\alpha_{1}-i T_{m},-m-1 / 2-\sigma-i T_{m},-m-1 / 2-$ $\sigma+i T_{m}, \alpha_{1}+i T_{m}$ where $m \geq 2$ is an integer and $T_{m}$ is the number defined in Lemma 3.5. We note that whenever $u+\sigma \geq(k+1) / 2+\varepsilon$,

$$
\left|\frac{L_{f}^{\prime}}{L_{f}}(s+w)\right| \ll 1
$$

and also from Lemma 3.5, the contributions from the horizontal portion and the vertical portion tend to zero as $m \rightarrow \infty$. Therefore the integral on the right hand side of (3.6.2) is equal to $2 \pi i$ times the sum of the residues of the integrand in the half plane $\Re w<\alpha_{1}$. The singularities are $w=0$, $w=-q-s(q=0,1,2, \ldots)$ and $w=\varrho-s$, and the corresponding residues are

$$
-(\log x)^{2} \frac{L_{f}^{\prime}}{L_{f}}(s), \quad \frac{x^{-q-s}\left(1-x^{-q-s}\right)^{2}}{(q+s)^{3}}, \quad \frac{x^{\varrho-s}\left(1-x^{\varrho-s}\right)^{2}}{(s-\varrho)^{3}}
$$

respectively. Since the series of the residues is absolutely convergent we get the lemma.

Lemma 3.7. Let $w=u+i v$. Then for $|v| \geq 10$, we have

$$
J_{1}=\int_{-\infty}^{\infty} \Psi_{U}(t / T) t^{w} d t \ll \frac{T^{u+1} U^{3}\left(1+U^{-1}\right)^{u+4}}{|(w+1)(w+2)(w+3) v|} .
$$

Proof. Integration by parts and the properties of $\Psi_{U}$ give

$$
\left|J_{1}\right| \leq \frac{T^{u+1}\left|\int_{-\infty}^{\infty} \Psi_{U}^{(3)}(t) t^{u+3+i v} d t\right|}{|(w+1)(w+2)(w+3)|} .
$$

Let $G(t)=\Psi_{U}^{(3)}(t) t^{u+3}, F(t)=v \log t$. Then

$$
\frac{G(t)}{F^{\prime}(t)}=v^{-1} \Psi_{U}^{(3)}(t) t^{u+4}
$$

is monotonic in $t$ in the interval $1+U^{-1} \leq t \leq 1+H T^{-1}-U^{-1}$. Also, for any $v>0$,

$$
\frac{F^{\prime}(t)}{G(t)} \geq v U^{-3}\left(1+U^{-1}\right)^{-u-4}>0
$$


since $\Psi_{U}^{(3)}(t) \ll U^{3}$. Hence by Lemma 4.3 of [14], we have

$$
\left|\int_{-\infty}^{\infty} \Psi_{U}^{(3)}(t) t^{u+3+i v} d t\right| \leq 4 v^{-1} U^{3}\left(1+U^{-1}\right)^{u+4} .
$$

This proves the lemma for $v>0$. For $v<0$ the proof is similar.

Lemma 3.8. For $|v| \leq 10$, we have

$$
J_{2}=\int_{-\infty}^{\infty} \Psi_{U}(t / T) t^{w} d t \ll \frac{T^{u+1}}{u+1}\left\{\left(2-U^{-1}\right)^{u+1}-\left(1+U^{-1}\right)^{u+1}\right\} .
$$

Proof. Using the properties of $\Psi_{U}$ and taking the absolute value inside the integral, a trivial estimation gives the lemma.

Lemma 3.9. If $U=4 T / H$, then for $k / 2 \leq \sigma \leq k / 2+\varepsilon$, we have

$$
\left|J_{3}\right|=\left|\int_{-\infty}^{\infty} \Psi_{U}(t / T)\left(\frac{t}{2 \pi}\right)^{2 k-4 \sigma} d t\right| \ll_{\varepsilon} H .
$$

Proof. By a suitable change of variable, we have

$$
\begin{aligned}
\left|J_{3}\right| & =\left|\left(\frac{T}{2 \pi}\right)^{2 k-4 \sigma} T \int_{-\infty}^{\infty} \Psi_{U}(t) t^{2 k-4 \sigma} d t\right| \\
& \leq \frac{T^{2 k-4 \sigma+1}}{(2 \pi)^{2 k-4 \sigma}} \int_{1+U^{-1}}^{1+H T^{-1}-U^{-1}} t^{2 k-4 \sigma} d t \\
& \ll{ }_{\varepsilon} T^{2 k-4 \sigma+1}(H / T-2 / U)\left(1+U^{-1}\right)^{2 k-4 \sigma} \ll H
\end{aligned}
$$

since $\sigma \geq k / 2$.

Lemma 3.10. For $b>0, \sigma>k / 2$, we have

$$
\sum_{v_{1}, v_{2} \leq \xi^{2}} \frac{\lambda_{v_{1}} \lambda_{v_{2}}}{\left(v_{1} v_{2}\right)^{\sigma}} \frac{\left(v_{1} v_{2}\right)^{b}}{\left(v_{1}, v_{2}\right)^{2 b}} \ll \xi^{4 b+2}(\log \xi)^{2} .
$$

Proof. Since $\left|\lambda_{v}\right|=\left|\mu_{f}(v) g_{\xi}(v)\right| \leq d(v) v^{(k-1) / 2}$, we have

$$
\begin{aligned}
\sum_{v_{1}, v_{2} \leq \xi^{2}} \frac{\lambda_{v_{1}} \lambda_{v_{2}}}{\left(v_{1} v_{2}\right)^{\sigma}} \frac{\left(v_{1} v_{2}\right)^{b}}{\left(v_{1}, v_{2}\right)^{2 b}} & \ll \sum_{v_{1}, v_{2} \leq \xi^{2}} d\left(v_{1}\right) d\left(v_{2}\right)\left(v_{1} v_{2}\right)^{b-1 / 2} \\
& \ll \xi^{4 b-2}\left(\sum_{v \leq \xi^{2}} d(v)\right)^{2} \ll \xi^{4 b+2}(\log \xi)^{2}
\end{aligned}
$$

because $\sigma>k / 2$, and this proves the lemma. 
LEMMA 3.11. We have

$$
\int_{T}^{T+H}\left|L_{f}(k / 2+1+i t) M_{\xi^{2}}(k / 2+1+i t)-1\right|^{2} d t \ll H / \xi^{2-\varepsilon} .
$$

Proof. We write

$$
L_{f}(s) M_{\xi^{2}}(s)-1=\sum_{n=1}^{\infty} c_{n} n^{-s}
$$

We note that $a_{n} * \mu_{f}(n)=I(n)=[1 / n]$ (the Dirichlet convolution) and hence $c_{n}=0$ for $2 \leq n \leq \xi$. Also we notice that, by definition, $c_{1}=1$ and for $n \geq \xi$,

$$
c_{n}=\sum_{d \mid n} a_{d} \mu_{f}(n / d) g_{\xi}(n / d)
$$

Therefore

$$
\left|c_{n}\right| \leq \sum_{m \mid n} d(m) m^{(k-1) / 2} d(n / m)(n / m)^{(k-1) / 2} \leq d_{4}(n) n^{(k-1) / 2}
$$

since $\left|\mu_{f}(n / d)\right| \leq a_{n / d},\left|g_{\xi}(n / d)\right| \leq 1$ and $\left|a_{m}\right| \leq d(m) m^{(k-1) / 2}$. Hence we obtain

$$
\left|c_{n}\right|^{2} \leq n^{k-1} d_{16}(n)
$$

From the Montgomery-Vaughan theorem (see [10]), on using (3.11.4) we get

$$
\begin{aligned}
J_{4} & :=\int_{T}^{T+H}\left|L_{f}(k / 2+1+i t) M_{\xi^{2}}(k / 2+1+i t)-1\right|^{2} d t \\
& =\sum_{n \geq \xi}\left|c_{n}\right|^{2} n^{-k-2}(H+O(n)) \\
& \ll H \sum_{\xi \leq n \leq H} n^{-3} d_{16}(n)+\sum_{n \geq H} n^{-2} d_{16}(n) \\
& \ll \frac{H(\log T)^{15}}{\xi^{2}}+\frac{(\log T)^{15}}{H} \ll \frac{H}{\xi^{2-\varepsilon}},
\end{aligned}
$$

which proves the lemma.

LEMMA 3.12. If $k / 2<\sigma<k / 2+1 / 1000$ and $\mu, \nu$ are co-prime positive integers $\leq T$, then

$$
J_{5}:=\int_{-\infty}^{\infty} \Psi_{U}(t / T)\left|L_{f}(\sigma+i t)\right|^{2}(\mu / \nu)^{i t} d t
$$




$$
\begin{aligned}
& =(\mu \nu)^{-\sigma} D_{\mu \nu}(2 \sigma) \int_{-\infty}^{\infty} \Psi_{U}(t / T) d t \\
& +(\mu \nu)^{-(k-\sigma)} D_{\mu \nu}(2(k-\sigma)) \int_{-\infty}^{\infty} \Psi_{U}(t / T)(t / T)^{2 k-4 \sigma} d t \\
& \quad+O\left(\frac{(\mu \nu)^{2876 / 1000} U^{4} T^{3 / 4+\varepsilon}}{2 \sigma-k}\right)
\end{aligned}
$$

where

$$
D_{\mu \nu}(s)=\sum_{l=1}^{\infty} \frac{a_{\mu l} a_{\nu l}}{l^{s}} .
$$

Proof. First consider the following expression:

$$
\begin{aligned}
& E=(\mu \nu)^{\sigma} \\
& \times \sum_{0 \leq l \leq \sqrt{\mu \nu} U T^{\varepsilon}} \frac{1}{2 \pi i} \int_{(2)}\left(\frac{\sqrt{\mu \nu}}{2 \pi}\right)^{s} H_{l}(s) D_{\mu \nu}(s+2 \sigma, l) \int_{-\infty}^{\infty} \Psi_{U}(t / T) t^{s} d t
\end{aligned}
$$

where

$$
H_{l}(w)=\int_{0}^{\infty} \phi(\xi) e^{2 \pi i(l / \sqrt{\mu \nu}) \xi^{-1}} \xi^{w-1} d \xi
$$

and

$$
D_{\mu \nu}(w+2 \sigma, l)=\sum_{n=1}^{\infty} \frac{a_{n} a_{(n \nu+l) / \mu}}{(n \nu+l / 2)^{w+2 \sigma}} .
$$

We move the line of integration in $(3.12 .1)$ to $\Re s=-1 / 4$. From Lemma 5 of [5], we have

$$
D_{\mu \nu}(w+2 \sigma, l)=O\left(\frac{l|w+2 \sigma|^{1+\varepsilon}}{(\mu \nu)^{k / 2-2}(u+2 \sigma-k+1 / 4)}\right)
$$

uniformly in $\mu, \nu, l \geq 1, u+2 \sigma \geq k-1 / 4$. Note that here $u=-1 / 4$. Using integration by parts and from the properties of $\phi(\xi)$, it follows that

$$
H_{l}(w) \ll \frac{\sqrt{\mu \nu}}{l}|w| .
$$

From Lemmas 3.7, 3.8 and the inequalities (3.12.4), (3.12.5) we obtain

$$
\begin{aligned}
E & \ll \frac{(\mu \nu)^{\sigma+u / 2+1 / 2+1 / 2}}{(2 \sigma-k)(\mu \nu)^{k / 2-2}} U^{4} T^{3 / 4+\varepsilon} \\
& \ll \frac{(\mu \nu)^{\sigma+23 / 8-k / 2}}{2 \sigma-k} U^{4} T^{3 / 4+\varepsilon} \ll \frac{(\mu \nu)^{2876 / 1000}}{2 \sigma-k} U^{4} T^{3 / 4+\varepsilon} .
\end{aligned}
$$

Now the lemma follows from Lemma 2.1 of [9]. 
Lemma 3.13. For $\sigma=k / 2+A_{8} / \log T$, with $U=4 T / H$ we have

$$
\int_{T}^{T+H}\left|L_{f}(\sigma+i t) M_{\xi^{2}}(\sigma+i t)-1\right|^{2} d t \ll H+O\left(\frac{T^{19 / 4+\varepsilon} \xi^{13.504}(\log \xi)^{3}}{H^{4}}\right) .
$$

Proof. From Lemmas 3.10 and 3.12, with $b=2876 / 1000$, we have

$$
\begin{aligned}
J_{6}:= & \int_{-\infty}^{\infty} \Psi_{U}(t / T)\left|L_{f}(\sigma+i t) M_{\xi^{2}}(\sigma+i t)-1\right|^{2} d t \\
= & \sum_{v_{1}, v_{2} \leq \xi^{2}} \frac{\lambda_{v_{1}} \lambda_{v_{2}}}{\left(v_{1} v_{2}\right)^{2 \sigma}}\left(v_{1}, v_{2}\right)^{2 \sigma} J_{7} \\
& +\sum_{v_{1}, v_{2} \leq \xi^{2}} \frac{\lambda_{v_{1}} \lambda_{v_{2}}}{\left(v_{1} v_{2}\right)^{k}}\left(v_{1}, v_{2}\right)^{2(k-\sigma)} J_{8} \\
& +O\left(T^{3 / 4+\varepsilon} U^{4} \xi^{13.504}(\log \xi)^{3}\right),
\end{aligned}
$$

where

$$
\begin{aligned}
& J_{7}:=D_{v_{1} /\left(v_{1}, v_{2}\right), v_{2} /\left(v_{1}, v_{2}\right)}(2 \sigma) \int_{-\infty}^{\infty} \Psi_{U}(t / T) d t \\
& J_{8}:=D_{v_{1} /\left(v_{1}, v_{2}\right), v_{2} /\left(v_{1}, v_{2}\right)}(2(k-\sigma)) \int_{-\infty}^{\infty} \Psi_{U}(t / T)(t / T)^{2 k-4 \sigma} d t .
\end{aligned}
$$

Also note that

$$
\begin{aligned}
\int_{-\infty}^{\infty} \Psi_{U}(t / T) d t & =T \int_{-\infty}^{\infty} \Psi_{U}(t) d t \\
& =T \int_{1+U^{-1}}^{1+H T^{-1}-U^{-1}} d t=T\left\{H T^{-1}-2 U^{-1}\right\} \ll H .
\end{aligned}
$$

Now the lemma follows from the arguments of Section 3 of [9].

LEMmA 3.14. Let $B$ be any small positive constant. For $T^{19 / 20+13.505 B / 5}$ $\ll H \leq T$, we have

$$
\int_{T}^{T+H}\left|L_{f}(\sigma+i t) M_{\xi^{2}}(\sigma+i t)-1\right|^{2} d t \ll_{\varepsilon} H T^{-(2-\varepsilon)(\sigma-k / 2) B}
$$

uniformly for $k / 2+A_{9} / \log T \leq \sigma \leq k / 2+1$.

Proof. We fix $\xi=T^{B}$ so that the error in Lemma 3.13 is

$$
\ll H^{-4} T^{19 / 4+13.504 B+\varepsilon}(\log T)^{3} \ll H^{-4} T^{19 / 4+13.504 B+\varepsilon_{1}} \ll H
$$


for some other small positive constant $\varepsilon_{1}(<0.001 B)$, since $T^{19 / 20+13.505 B / 5}$ $\ll H$. Hence from Lemma 3.13 we have

$$
\int_{T}^{T+H}\left|L_{f}\left(k / 2+A_{9} / \log T+i t\right) M_{\xi^{2}}\left(k / 2+A_{9} / \log T+i t\right)-1\right|^{2} d t \ll H .
$$

Also from Lemma 3.11, we have

$$
\int_{T}^{T+H}\left|L_{f}(k / 2+1+i t) M_{\xi^{2}}(k / 2+1+i t)-1\right|^{2} d t \ll H T^{-B(2-\varepsilon)} .
$$

Now we use the two-variable Gabriel convexity theorem (see [4]):

ConveXity TheOREm. Let $g(s)$ be an analytic function in a specified region and for any positive $\lambda$, let

$$
G(\sigma, \lambda)=\left(\int_{T}^{T+H}|g(\sigma+i t)|^{1 / \lambda} d t\right)^{\lambda} .
$$

Then, for $\alpha<\sigma<\beta$ and any positive numbers $\lambda, \mu$, with $p=(\beta-\sigma) /(\beta-\alpha)$ and $q=(\sigma-\alpha) /(\beta-\alpha)$, we have

$$
G(\sigma, \lambda p+\mu q) \ll(G(\alpha, \lambda))^{p}(G(\beta, \mu))^{q} .
$$

In the above convexity theorem, we take $\lambda=\mu=1 / 2, \alpha=k / 2+$ $A_{9} / \log T, \beta=k / 2+1$ and $g(s)=L_{f}(s) M_{\xi^{2}}(s)-1$. This implies that

$$
p=\frac{k / 2+1-\sigma}{1-A_{9} / \log T}, \quad q=\frac{\sigma-k / 2-A_{9} / \log T}{1-A_{9} / \log T} .
$$

Note that $p+q=1$. From (3.14.2), (3.14.3) and (3.14.5), we obtain

$$
\begin{aligned}
& \int_{T}^{T+H}\left|L_{f}(\sigma+i t) M_{\xi^{2}}(\sigma+i t)-1\right|^{2} d t \\
& \quad \ll H^{p}\left(H T^{-B(2-\varepsilon)}\right)^{q} \ll H T^{-B q(2-\varepsilon)} \ll H T^{-B(2-\varepsilon)(\sigma-k / 2)}
\end{aligned}
$$

and hence the lemma.

REMARK. Let $B^{\prime}=19 / 20+13.505 B / 5$ where $B$ is any fixed small positive constant. Let $T^{\alpha} \leq H \leq T, B^{\prime}<\alpha \leq 1$. We notice that

$$
\left(\frac{H}{T^{B^{\prime}}}\right) \leq T^{1-B^{\prime}} \text { and hence }\left(\frac{H}{T^{B^{\prime}}}\right)^{1 /\left(1-B^{\prime}\right)} \leq T \text {. }
$$

Therefore, we have

$$
T^{-(2-\varepsilon) B(\sigma-k / 2)} \leq T^{-B(\sigma-k / 2)} \leq\left(\frac{H}{T^{B^{\prime}}}\right)^{-\frac{B}{1-B^{\prime}}(\sigma-k / 2)} .
$$


This implies that, for $T^{\alpha} \leq H \leq T$ with $B^{\prime}<\alpha \leq 1$,

$$
\int_{T}^{T+H}\left|L_{f}(\sigma+i t) M_{\xi^{2}}(\sigma+i t)-1\right|^{2} d t \ll H\left(\frac{H}{T^{B^{\prime}}}\right)^{-\frac{B}{1-B^{\prime}}(\sigma-k / 2)},
$$

which holds uniformly for $k / 2+A_{10} / \log \xi \leq \sigma \leq k / 2+1 / 2$.

4. Proof of Theorem 1. We follow closely the arguments of Selberg (see [13]). For $x \geq 2, t>0$, we define a number

$$
\sigma_{x, t}=\frac{k}{2}+2 \max _{\varrho}\left(\beta-\frac{k}{2}, \frac{2}{\log x}\right)
$$

where $\varrho$ runs through all zeros $\varrho=\beta+i \gamma$ for which

$$
|t-\gamma| \leq \frac{x^{3|\beta-k / 2|}}{\log x}
$$

We notice that

$$
\sum_{\varrho} \frac{\beta}{\beta^{2}+\gamma^{2}}=O(\log t)
$$

and hence from Lemma 3.2, for $t \geq 2$, taking real parts on both sides, we obtain

$$
S:=\sum_{\varrho} \frac{\sigma_{x, t}-\beta}{\left(\sigma_{x, t}-\beta\right)^{2}+(t-\gamma)^{2}}=\Re \frac{L_{f}^{\prime}}{L_{f}}\left(\sigma_{x, t}+i t\right)+O(\log t) .
$$

Since zeros lie symmetrically with respect to the line $\sigma=k / 2$, we have

$$
\begin{aligned}
S= & \left(\sigma_{x, t}-k / 2\right) \\
& \times \sum_{\varrho} \frac{\left\{\left(\sigma_{x, t}-k / 2\right)^{2}-(\beta-k / 2)^{2}+(t-\gamma)^{2}\right\}}{\left\{\left(\sigma_{x, t}-\beta\right)^{2}+(t-\gamma)^{2}\right\}\left\{\left(\sigma_{x, t}-k+\beta\right)^{2}+(t-\gamma)^{2}\right\}} .
\end{aligned}
$$

Arguing as in [13], we find that

$$
\begin{aligned}
S_{1} & :=\left(\sigma_{x, t}-\frac{k}{2}\right)^{2}-\left(\beta-\frac{k}{2}\right)^{2}+(t-\gamma)^{2} \\
& \geq \frac{3}{10}\left\{\left(\sigma_{x, t}-\beta\right)^{2}+\left(\sigma_{x, t}-k+\beta\right)^{2}+2(t-\gamma)^{2}\right\} .
\end{aligned}
$$

Therefore from (4.4) and (4.5), we get

$$
\begin{aligned}
S \geq & \frac{3}{10}\left(\sigma_{x, t}-k / 2\right) \\
& \times \sum_{\varrho}\left\{\frac{1}{\left(\sigma_{x, t}-\beta\right)^{2}+(t-\gamma)^{2}}+\frac{1}{\left(\sigma_{x, t}-k+\beta\right)^{2}+(t-\gamma)^{2}}\right\} \\
= & \frac{3}{5}\left(\sigma_{x, t}-k / 2\right) \sum_{\varrho} \frac{1}{\left(\sigma_{x, t}-\beta\right)^{2}+(t-\gamma)^{2}} .
\end{aligned}
$$


From (4.3) and (4.6) we have

$$
\begin{aligned}
\sum_{\varrho} \frac{1}{\left(\sigma_{x, t}-\beta\right)^{2}+(t-\gamma)^{2}}< & \frac{5}{3} \frac{1}{\sigma_{x, t}-k / 2}\left|\frac{L_{f}^{\prime}}{L_{f}}\left(\sigma_{x, t}+i t\right)\right| \\
& +O\left(\frac{\log t}{\sigma_{x, t}-k / 2}\right) .
\end{aligned}
$$

For $t \geq 2,2 \leq x \leq t^{2}, \sigma \geq \sigma_{x, t}$, Lemma 3.6 yields

$$
\begin{aligned}
\frac{L_{f}^{\prime}}{L_{f}}(s)= & -\sum_{n<x^{3}} \frac{\Lambda_{x, f}(n)}{n^{s}}+O\left(x^{-\sigma}(\log x)^{-2}\right) \\
& +\frac{\omega}{(\log x)^{2}} \sum_{\varrho} \frac{x^{\beta-\sigma}\left(1+x^{\beta-\sigma}\right)^{2}}{\left\{(\sigma-\beta)^{2}+(t-\gamma)^{2}\right\}^{3 / 2}}
\end{aligned}
$$

where $|\omega| \leq 1$.

Now, arguing as in [13], we obtain

$$
\frac{x^{\beta-\sigma}\left(1+x^{\beta-\sigma}\right)^{2}}{\left\{(\sigma-\beta)^{2}+(t-\gamma)^{2}\right\}^{3 / 2}}<\frac{2(\log x) x^{k / 4-\sigma / 2}}{\left(\sigma_{x, t}-\beta\right)^{2}+(t-\gamma)^{2}} .
$$

Therefore from (4.7) and (4.9), we get

$$
\begin{aligned}
\sum_{\varrho} \frac{x^{\beta-\sigma}\left(1+x^{\beta-\sigma}\right)^{2}}{\left\{(\sigma-\beta)^{2}+(t-\gamma)^{2}\right\}^{3 / 2}}< & \frac{5}{6}(\log x)^{2} x^{k / 4-\sigma / 2}\left|\frac{L_{f}^{\prime}}{L_{f}}\left(\sigma_{x, t}+i t\right)\right| \\
& +O\left(x^{k / 4-\sigma / 2}(\log x)^{2} \log t\right) .
\end{aligned}
$$

Hence from (4.10), (4.8) becomes

$$
\begin{aligned}
\frac{L_{f}^{\prime}}{L_{f}}(s)= & -\sum_{n<x^{3}} \frac{\Lambda_{x, f}(n)}{n^{s}}+O\left(x^{k / 4-\sigma / 2} \log t\right) \\
& +\frac{5}{6} \omega^{\prime} x^{k / 4-\sigma / 2} \frac{L_{f}^{\prime}}{L_{f}}\left(\sigma_{x, t}+i t\right)
\end{aligned}
$$

where $\left|\omega^{\prime}\right|<1$. Taking first $\sigma=\sigma_{x, t}$, we get

$$
\frac{L_{f}^{\prime}}{L_{f}}\left(\sigma_{x, t}+i t\right)=O\left(\left|\sum_{n<x^{3}} \frac{\Lambda_{x, f}(n)}{n^{\sigma_{x, t}+i t}}\right|\right)+O(\log t) .
$$

Therefore from (4.7) and (4.12), we get

$$
\sum_{\varrho} \frac{\sigma_{x, t}-k / 2}{\left(\sigma_{x, t}-\beta\right)^{2}+(t-\gamma)^{2}}=O\left(\left|\sum_{n<x^{3}} \frac{\Lambda_{x, f}(n)}{n^{\sigma_{x, t}+i t}}\right|\right)+O(\log t)
$$

and

$$
\begin{aligned}
\frac{L_{f}^{\prime}}{L_{f}}(s)= & -\sum_{n<x^{3}} \frac{\Lambda_{x, f}(n)}{n^{s}}+O\left(x^{k / 4-\sigma / 2}\left|\sum_{n<x^{3}} \frac{\Lambda_{x, f}(n)}{n^{\sigma_{x, t}+i t}}\right|\right) \\
& +O\left(x^{k / 4-\sigma / 2} \log t\right) .
\end{aligned}
$$


Now,

$$
\begin{aligned}
\arg L_{f}(k / 2+i t)= & -\int_{k / 2}^{\infty} \Im \frac{L_{f}^{\prime}}{L_{f}}(\sigma+i t) d \sigma \\
= & \int_{\sigma_{x, t}}^{\infty} \Im \frac{L_{f}^{\prime}}{L_{f}}(\sigma+i t) d \sigma-\left(\sigma_{x, t}-k / 2\right) \Im \frac{L_{f}^{\prime}}{L_{f}}\left(\sigma_{x, t}+i t\right) \\
& +\int_{k / 2}^{\sigma_{x, t}} \Im\left\{\frac{L_{f}^{\prime}}{L_{f}}\left(\sigma_{x, t}+i t\right)-\frac{L_{f}^{\prime}}{L_{f}}(\sigma+i t)\right\} d \sigma \\
= & I_{1}+I_{2}+I_{3} \text { (say). }
\end{aligned}
$$

Using (4.14), we find that

$$
I_{1}=\Im \sum_{n<x^{3}} \frac{\Lambda_{x, f}(n)}{n^{\sigma_{x, t}+i t} \log n}+O\left(\frac{1}{\log x}\left|\sum_{n<x^{3}} \frac{\Lambda_{x, f}(n)}{n^{\sigma_{x, t}+i t}}\right|\right)+O\left(\frac{\log t}{\log x}\right) .
$$

From (4.12), we get

$$
I_{2}=O\left(\left(\sigma_{x, t}-k / 2\right)\left|\sum_{n<x^{3}} \frac{\Lambda_{x, f}(n)}{n^{\sigma_{x, t}+i t}}\right|\right)+O\left(\left(\sigma_{x, t}-k / 2\right) \log t\right) .
$$

From Lemma 3.2, taking the imaginary part of both sides and arguing as in [13], we find that

$$
\begin{aligned}
\left|I_{3}\right| & <10\left(\sigma_{x, t}-k / 2\right) \sum_{\varrho} \frac{\sigma_{x, t}-k / 2}{\left(\sigma_{x, t}-\beta\right)^{2}+(t-\gamma)^{2}}+O\left(\left(\sigma_{x, t}-k / 2\right) \log t\right) \\
& =O\left(\left(\sigma_{x, t}-k / 2\right)\left|\sum_{n<x^{3}} \frac{\Lambda_{x, f}(n)}{n^{\sigma_{x, t}+i t}}\right|\right)+O\left(\left(\sigma_{x, t}-k / 2\right) \log t\right) .
\end{aligned}
$$

Now Theorem 1 follows from (4.15)-(4.18).

5. Proof of Theorem 2. It suffices to show that (for any fixed small positive constant $B, T^{\alpha} \leq H \leq T, B^{\prime}<\alpha \leq 1$ where $B^{\prime}$ is as in the theorem)

$$
\int_{\sigma}^{(k+1) / 2}\left(N_{f}\left(\sigma^{\prime}, T+H\right)-N_{f}\left(\sigma^{\prime}, T\right)\right) d \sigma^{\prime} \ll H\left(\frac{H}{T^{B^{\prime}}}\right)^{-\frac{B}{1-B^{\prime}}(\sigma-k / 2)}
$$

for $k / 2+A_{11} / \log \xi \leq \sigma \leq(k+1) / 2$.

Let $\Phi(s)=1-\left(L_{f}(s) M_{\xi^{2}}(s)-1\right)^{2}$. The zeros of $L_{f}(s)$ occur among those of $\Phi(s)$ with at least the same multiplicities. By Littlewood's lemma regarding the number of zeros of an analytic function in a rectangle (see 
$[15])$, we obtain

$$
\begin{aligned}
& \int_{\sigma}^{(k+1) / 2}\left(N_{f}\left(\sigma^{\prime}, T+H\right)-N_{f}\left(\sigma^{\prime}, T\right)\right) d \sigma^{\prime} \\
& \leq \frac{1}{2 \pi} \int_{T}^{T+H} \log |\Phi(\sigma+i t)| d t \\
& \quad+\frac{1}{2 \pi} \int_{\sigma}^{\infty} \arg \Phi\left(\sigma^{\prime}+i(T+H)\right) d \sigma^{\prime}-\frac{1}{2 \pi} \int_{\sigma}^{\infty} \arg \Phi\left(\sigma^{\prime}+i T\right) d \sigma^{\prime} .
\end{aligned}
$$

In the range $((k+1) / 2+4, \infty)$, we see that $\arg \Phi\left(\sigma^{\prime}+i t\right)=O\left(2^{-\sigma}\right)$ and hence

$$
\int_{(k+1) / 2+4}^{\infty} \arg \Phi\left(\sigma^{\prime}+i T\right) d \sigma^{\prime}=O(1) .
$$

In the range $(k / 2,(k+1) / 2+4)$, from Jensen's theorem (see [14]) and a standard argument, we find that

$$
\arg \Phi\left(\sigma^{\prime}+i T\right)=O(\log T) .
$$

Therefore we get

$$
\int_{\sigma}^{\infty} \arg \Phi\left(\sigma^{\prime}+i T\right) d \sigma^{\prime} \ll \log T .
$$

Similarly we have

$$
\int_{\sigma}^{\infty} \arg \Phi\left(\sigma^{\prime}+i(T+H)\right) d \sigma^{\prime} \ll \log T .
$$

Since $\log (1+|x|) \leq|x|$, we obtain

$$
\begin{aligned}
\int_{T}^{T+H} \log |\Phi(\sigma+i t)| d t & \leq \int_{T}^{T+H}\left|L_{f}(\sigma+i t) M_{\xi^{2}}(\sigma+i t)-1\right|^{2} d t \\
& \ll H\left(\frac{H}{T^{B^{\prime}}}\right)^{-\frac{B}{1-B^{\prime}}(\sigma-k / 2)} .
\end{aligned}
$$

Now the inequality (5.1) follows from (5.2) to (5.7). Hence it is enough to assume that $\sigma-k / 2 \geq(\log T)^{-1}$. Therefore,

$$
\begin{aligned}
N_{f}(\sigma, T, T+H) & \leq(\log T) \int_{\sigma-1 / \log T}^{\sigma}\left\{N_{f}\left(\sigma^{\prime}, T+H\right)-N_{f}\left(\sigma^{\prime}, T\right)\right\} d \sigma^{\prime} \\
& \ll H\left(\frac{H}{T^{B^{\prime}}}\right)^{-\frac{B}{1-B^{\prime}}(\sigma-k / 2)} \log T
\end{aligned}
$$

from (5.7) and this proves Theorem 2. 


\section{References}

[1] E. Bombieri and D. A. Hejhal, On the distribution of zeros of linear combinations of Euler products, Duke Math. J. 80 (1995), 821-862.

[2] P. Deligne, Formes modulaires et représentations l-adiques, Sém. Bourbaki, 1968/69, exposés 355.

[3] —, La conjecture de Weil, I, Inst. Hautes Études Sci. Publ. Math. 43 (1974), 273307.

[4] R. M. Gabriel, Some results concerning the integrals of moduli of regular functions along certain curves, J. London Math. Soc. 2 (1927), 112-117.

[5] J. L. Hafner, Zeros on the critical line for Dirichlet series attached to certain cusp forms, Math. Ann. 264 (1983), 21-37.

[6] G. H. Hardy, Note on Ramanujan's function $\tau(n)$, Proc. Cambridge Philos. Soc. 23 (1927), 675-680.

[7] E. Hecke, Über die Bestimmung Dirichletscher Reihen durch ihre Funktionalgleichung, Math. Ann. 112 (1936), 664-669.

[8] A. E. Ingham, The Distribution of Prime Numbers, edited by G. H. Hardy and E. Cunningham, Stechert-Hafner Service Agency, New York-London, 1964.

[9] W. Luo, Zeros of Hecke L-functions associated with cusp forms, Acta Arith. 71 (1995), 139-158.

[10] H. L. Montgomery and R. C. Vaughan, Hilbert's inequality, J. London Math. Soc. 8 (1974), 73-82.

[11] R. A. Rankin, Contributions to the theory of Ramanujan's function $\tau(n)$ and similar arithmetic functions, Proc. Cambridge Philos. Soc. 35 (1939), 357-372.

[12] A. Selberg, On the zeros of Riemann's zeta-function, in: Collected Papers, Vol. I, Springer, 1989, 85-141.

[13] - Contributions to the theory of the Riemann zeta-function, in: Collected Papers, Vol. I, Springer, 1989, 214-280.

[14] E. C. Titchmarsh, The Theory of the Riemann Zeta-Function, 2nd ed., revised by D. R. Heath-Brown, Oxford Univ. Press, 1986.

[15] —, The Theory of Functions, Oxford, 1952.

School of Mathematics

TIFR

Mumbai 400 005, India

E-mail: sank@math.tifr.res.in

Received on 1.6.2001

and in revised form on 10.6.2002 